\title{
Spectrum of Conversion Disorders: Clinical Perspective for a Neurologist
}

\author{
Gourav Goyal ${ }^{1}$, Surbhi Chaturvedi ${ }^{2}$
}

\begin{abstract}
Conversion disorders are a borderland between psychiatrist and neurologist. These are known from ancient time and witnessed a variety of nomenclature and theories. Manifestations of conversion disorders are widely variable and may range from psychogenic movement disorders, psychogenic sensorimotor deficit to nonepileptic psychogenic seizures. Though advancement in diagnostic techniques helps in diagnosis of a number of neurological disorders, still diagnosis of conversion disorders is mostly clinical. For a neurologist, it is important to know breadth of conversion disorders and salient clinical features to differentiate from organic disorders to avoid fallacious diagnosis and its serious consequences. Treatment options include psychotherapy, hypnotic therapy, pharmacotherapy and recently transcranial magnetic stimulation. All patients of conversion disorders may not have mild course.
\end{abstract}

Keywords: Conversion disorder, Neurologist, Psychiatrist

Journal of Mahatma Gandhi University of Medical Sciences and Technology (2018): 10.5005/jp-journals-10057-0068

\section{INTRODUCTION}

Conversion disorders witnessed a colorful history for long time. Prior to 16th century, the hysteria was linked with anillness of the uterus $^{1}$ or to explanations such as witchcraft or demonic possession. By the early 17th century, psychological basis of hysteria was postulated. Later, Freud suggested that the emotional charge of painful experiences would be consciously repressed as a way of managing the pain, but this emotional charge would be somehow "converted" into the neurological symptoms. ${ }^{2}$ Till mid of last century, psychological theories were well established for conversion disorders. Recently, newer imaging modalities provide new insight to understanding of neurobiological basis of conversion disorders. InDSM- IV TR, ${ }^{3}$ conversion disorders have been kept as a different category. Conversion disorders may have varied manifestations ranging from psychogenic movement disorders, nonepileptic psychogenic seizures to sensorimotor deficit. In an outpatient clinic, they may mimic a number of neurological disorders. Without knowing the spectrum of these disorders, they may be misdiagnosed, and can lead to wrong diagnosis and therapies. On other hand, some of neurological disorders may mimic conversion disorders and can lead to delay in diagnosis and further serious consequences. It is very important to know spectrum of conversion disorders and differentiation from organic neurological disorders. Here, in this article we have tried to highlight the important conversion disorders that can help in day to day neurology practice.

\section{Definition and DSM IV Criteria of Conversion Disorders}

Conversion disorder is a motor-sensory deficit after a stressful event without an organic etiology. ${ }^{3}$ Historically, conversion disorder is described as patient exhibiting "la belle indifference," a characteristic lack of normal concern about their deficit. ${ }^{4}$ Conversion disorders may be confused with somatization disorder; however, conversion disorder is distinguished by its relatively acute nature and narrower symptom complex. The American Psychiatric Association DSM-IV-TR defines diagnostic criteria for a conversion disorder (Box 1).
${ }^{1}$ Associate Professor, ${ }^{2}$ Senior Resident

1,2Department of Neurology, Mahatma Gandhi Medical College and Hospital, Jaipur, Rajasthan, India

Corresponding Author: Gourav Goyal, Associate Professor, Department of Neurology, Mahatma Gandhi Medical College and Hospital, Jaipur, Rajasthan, India, e-mail: drgauravjaipur@gmail.com

How to cite this article: Goyal G, Chaturvedi S. Spectrum of Conversion Disorders: Clinical Perspective for a Neurologist. J Mahatma Gandhi Univ Med Sci Tech 2018;3(1):7-16.

Source of support: Nil

Conflict of interest: None

Box 1: American Psychiatric Association DSM-IV-TR Diagnostic Criteria for a "Conversion Disorder" ${ }^{3}$

- At least 1 symptom or deficit of sensory or voluntary motor function suggesting neurologic or general medical condition.

- The symptoms are not limited to pain or sexual dysfunction, do not occur exclusively during the course of somatization disorder and can not be explained by another mental disorder.

- Appropriate study does not identify a neurologic or general medical condition or the direct effects of substance use that can fully explain symptoms.

- A stressful event precedes the onset of symptoms, suggesting that psychologic factors are involved.

- The patient does not consciously or intentionally feign the symptoms to assume a voluntary sick role (factitious disorder) or for secondary gain (malingering).

- It is not a culturally sanctioned behavior or experience.

- The condition warrants medical evaluation, causes clinically significant distress, or impairs social, occupational, or personal functioning.

\section{Dimension of Conversion Disorders}

Conversion disorders may have varied manifestations ranging from motor or sensory deficit to psychogenic movement disorders to

() The Author(s). 2018Open Access This article is distributed under the terms of the Creative Commons Attribution 4.0 International License (https://creativecommons. org/licenses/by-nc/4.0/), which permits unrestricted use, distribution, and non-commercial reproduction in any medium, provided you give appropriate credit to the original author(s) and the source, provide a link to the Creative Commons license, and indicate if changes were made. The Creative Commons Public Domain Dedication waiver (http://creativecommons.org/publicdomain/zero/1.0/) applies to the data made available in this article, unless otherwise stated. 
Box 2: Conversion disorders discussed in this review

- Psychogenic movements disorders
- Psychogenic dystonia
- Psychogenic tremor
- Psychogenic myoclonus
- Psychogenic parkinsonism
- Psychogenic sensorimotor deficit
- Nonepileptic psychogenic seizures

nonepileptic seizures. In Box 2, different conversion disorders has been listed that will be discussed individually in this review.

\section{Psychogenic Movement Disorders}

Psychogenic movement disorders are amongst the most frequent psychogenic symptoms. ${ }^{5}$ Psychogenic movement disorders include dystonia, tremor, myoclonus, tics, hemiballismus, chorea, parkinsonism and a host of bizarre gait and stance disturbances. Psychogenicdisorders that eventually turned out to be a physical illness has been reported in $6-30 \%$ of the patients. ${ }^{6}$ The diagnosis of idiopathic movement disorders can be done just by history and physical examination. Absence of a specific diagnostic laboratory test or any neuroimaging findings makes idiopathic movement disorders more susceptible to wrong diagnosis or its delay in diagnosis. To further complicate the matter, organic neurologic disease has been reported in as many as $28 \%$ of psychogenic neurologic disorders. ${ }^{7}$

\section{Diagnosis of Psychogenic Movement Disorders}

Diagnosis of psychogenic movement disorders must be made by a neurologist based on neurologic observations, though psychiatric analysis is essential. A comprehensive history and neurological examination as well as appropriate diagnostic studies (including MR imaging of the neuraxis, serum copper and ceruloplasmin levels, thyroid function test, parathyroid hormone or cerebrospinal fluid analysis) should be performed in proper clinical setting to exclude an organic etiology.

Fann and Williams proposed four levels of certainty for the diagnosis of psychogenic dystonia which can be applied to all types of movement disorders which are summarized in Table $1 .{ }^{8}$ Other diagnostic criteria have been proposed by Shill-Gerber ${ }^{9}$ and revision of Fann-Williams criteria by Gupta and Lang. ${ }^{10}$ The Shill-Gerber criterion haslacune as it gives more importance towards historical information rather than clinical characteristics of movement disorders and so has been criticized. Gupta and Lange proposed the revision of Fann-Williams criterion by making a new category of clinically definite by merging documented and clinically established categories and laboratory supported definite.
Box 3: Specific clinical features of psychogenic movement disorders (PMD)

- Abrupt onset and static course
- Inconsistent character of movements ( amplitude, frequency,
distribution, selective disability)
- Unresponsiveness to appropriate medications and responsive-
ness to placebos
- Movements increasing with attention and decreasing with
- Improvement with psychotherapy
- Diagnosed psychopathology

Box 4: Clinical aspects of idiopathic dystonia which help to differentiate from psychogenic dystonia

- Dystonia may begin in any part of the body and may spread over time.

- Generalized dystonia are usually childhood onset and develops gradually over years.

- Idiopathic dystonia usually begins with a specific action, though may be spontaneous.

- Use of sensory tricks to reduce dystonic postures.

- Dystonic movements usually remit with relaxation, hypnosis, and sleep and worsen by action.

- Sometime may manifest as "paroxysmal dystonia."

- Dystonic movements may have worsening in the evening.

- Dystonia rarely remits.

They have questioned the possible category. In this recent revision, importance to electrophysiological diagnosis of PMD has been given.

Specific psychogenic movement disorders: Despite the common guidelines for diagnosis of all PMD, some features of the motor symptoms and diagnosis are unique to the particular movement (Box 3).

\section{Psychogenic Dystonia}

Dystonia is characterized by sustained muscle contractions of agonist and antagonist muscles resulting in repetitive twisting and abnormal postures. Approximately two thirds of these patients suffer from idiopathic form. ${ }^{11}$ Hence, the correct diagnosis of psychogenic dystonia may be very difficult and susceptible to diagnostic errors in the absence of diagnostic test for organic dystonia. In most centres and almost all studies, psychogenic dystonia is the second most commonly encountered psychogenic movement disorder except for one study by Columbia University where it was the most frequent. ${ }^{12}$ Some of the features of organic dystonia are described in Box 4 to differentiate from psychogenic dystonia.

Table 1: Levels of certainty for the diagnosis of psychogenic movement disorders (PMD) ${ }^{8}$
1. Documented PMD
Movements are relieved by psychotherapy, psychological suggestion, administration of placebos and the patient must be witnessed to be free of symptoms when supposedly unobserved.
2. Clinically established PMD
Movements are inconsistent over time or incongruent with the classical symptomatology (i.e. a patient complaining of posturing of the limbs resists passive and active movement but easily grooms himself daily). In addition, one or more of the following present: other neurological signs that are definitely psychogenic (false weakness or sensory finding and self inflicted injuries), multiple somatizations or a documented psychiatric illness.

3. Probable PMD The movements are inconsistent or incongruent with the classical disorder but other features in support of psychogenicity are lacking.

4. Possible PMD A suspicion for a psychogenic basis for the movements is based only on the presence of an obvious emotional disturbance. 
Spectrum of Conversion Disorders: Clinical Perspective for a Neurologist

Table 2: Difference between organic and psychogenic tremor ${ }^{12}$

\begin{tabular}{lll}
\hline Features & Organic tremor & Psychogenic tremor \\
\hline Onset & Insidious & Sudden \\
Postural variation & Amplitude varies with different & Amplitudes remain same in all \\
& postures & postures \\
Clinical inconsistencies & No & Yes \\
Distractibility & May worse with distraction & Lessens \\
Attention & May reduce the amplitude & Increased \\
Changing pattern & Rare & Usual \\
Spontaneous remission & Rare & Usual \\
Response to anti-tremor drugs & Yes & No \\
Remission with psychotherapy & No & Yes \\
\hline
\end{tabular}

Box 5: Psychogenic mycolonus

The psychogenic mycolonus is characterized by following features:

- Abrupt onset and spontaneous remission

- Inconsistent character ( amplitude, frequency and distribution).

- Associated psychosocial stressors and psychopathology

- Marked reduction with distraction and worsen with attention.

- Exacerbation with suggestion and relief with placebo.

Knowledge of clinical features of organic dystonia is helpfu to differentiate it from psychogenic dystonia. Features like sudden onset, fixed posture since begging,excessive pain, associated bladder/bowel symptoms, response to placebo, lack of "Gesteantagoniste" and other associated functional motor deficit or psychogenic sensory loss favor psychogenic etiology of dystonia. Over diagnosis of psychogenic dystonia should be avoided as these have been reported only in $2.6 \%$ of the dystonias in one study. ${ }^{8}$

\section{Psychogenic Tremor}

Tremor is defined as a rhythmical, bidirectional oscillating movement owing to contraction of antagonist muscles that may occur at rest, with posture, or with movement. Psychogenic tremors have been reported to be the most common type of PMD, accounting for approximately $50 \%$ of the patients. ${ }^{13}$ Difference between organic and psychogenic tremor is summarized in Table $2 .^{12}$ Important differentiating points are fluctuation in amplitudes, frequency, involved body part, muscle activation and decreases by distracting the patient.

\section{Psychogenic Myoclonus}

Myoclonus is defined as sudden, brief, shock-like movement caused by either muscle contraction or by lapses in posture. Psychogenic myoclonus have been reported as the most common type of nonorganic movement disorder, accounting for $8.5 \%$ of all myoclonus and $20.2 \%$ of all psychogenic movement disorders in one movement disorders clinic. ${ }^{14}$ Features of Psychogenic myoclonus are summarized in Box $5 .^{14}$

\section{Psychogenic Parkinsonism}

Parkinsonism is a symptom complex of resting tremor, rigidity, bradykinesia, and impaired postural reflex. The clinical presentation of parkinsonism may varyand no definitive diagnostic test exists. Some salient features are highlighted in Table 3 which may help to differentiate psychogenic parkinsonism. ${ }^{15}$ This differentiation is challenging and requires considerable experience.

\section{Psychogenic Paralysis}

Psychogenic paralysis is characterized by loss of motor and sensory function subsequent to a psychiatric stressor. ${ }^{16}$ Associated symptoms can include loss of vision or hearing, pseudo-seizures, ataxia, and genitourinary and gastrointestinal dysfunction. ${ }^{17}$ The severity of motor symptoms generally exceeds that of sensory loss; rectal tone and reflexes are usually retained. ${ }^{18}$ In contrast, in a small study it was seen that diminished rectal tone, diminished rectal sensation, or diminished deep tendon reflexes were present in 12 of 14 patients with psychogenic paralysis. However, bulbo cavernosus reflex was retained in all. ${ }^{19}$ Rectal tone, rectal sensation and deep tendon reflexes may be subjected to a degree of voluntary control. The bulbocavernosus reflex is involuntary. These facts explain the findings. Recovery of psychogenic paralysis is rapid and complete, with more than $90 \%$ of patients showing complete recovery within 1 month. . $^{17,18}$

The diagnosis of a psychiatric disorder is of exclusion. When examining a patient with paralysis, clinical findings like normal reflexes, normal rectal sensation and tone, normal bulbocavernosus reflex should raise the suspicion for a psychologic etiology. Appropriate investigations should be done to exclude any organic etiology.

\section{Physical Examination for Psychogenic Paralysis}

There are number of physical examination maneuvers that have been validated to aid in the identification of psychogenic paralysis (Table 4).

\section{Spinal Injuries Center Test}

The Spinal Injuries Center (SIC) Test was described by Yugue et al for the evaluation of conversion disorder with motor deficit involving the lower extremities. ${ }^{20}$ In this test the patient is asked to be in the supine position and the examiner passively lifts the patient's knees to a flexed position with feet flat on the bed. The examiner releases the patient's knees; if the patient is able to maintain the flexed position, he is considered to be SIC test-positive. If the patient has severe paralysis, he won't be able to maintain his knees in the flexed position, and the leg on the paretic side will spontaneously fall into the flaccid extension position. In this scenario, the SIC test result is considered negative.

\section{Barre's Sign}

Barre's sign may be used for the evaluation of hemiplegia. The patient is placed in the prone position with knees flexed at right angles. The examiner releases the knees and asks the patient to maintain the position; the limb on the affected side will fall into extension in the event of true paralysis. In a patient with psychogenic hemiparalysis, the affected limb will extend rapidly without contraction of the hamstrings. ${ }^{20}$ 
Table 3: Clues suggesting psychogenic parkinsonism ${ }^{15}$

\begin{tabular}{|c|c|c|}
\hline Feature & Psychogenic parkinsonism & Organic parkinsonism \\
\hline Onset & Often sudden & Gradual \\
\hline Course & Static, maximum disability early & Progressive \\
\hline Laterality & Dominant side most affected & Any side may be affected \\
\hline Tremor & May be of any type (Rest, postural, action) & Usually resting tremor \\
\hline Variability & $\begin{array}{l}\text { Reduces with distraction/concentration, } \\
\text { increases with attention }\end{array}$ & Worsen with concentration \\
\hline Rigidity & $\begin{array}{l}\text { "voluntary resistance", cogwheeling absent, } \\
\text { may decrease with distraction }\end{array}$ & Cog wheeling present \\
\hline Bradykinesia & $\begin{array}{l}\text { No true fatiguing, marked slowness, bizarre } \\
\text { features. }\end{array}$ & $\begin{array}{l}\text { Worsen with time, may cause significant } \\
\text { fatiguing }\end{array}$ \\
\hline Gait & $\begin{array}{l}\text { Atypical, arm held stiffly at side, analgic if } \\
\text { pain associated. }\end{array}$ & $\begin{array}{l}\text { Short, shuffling gait, tendency to lean } \\
\text { forward }\end{array}$ \\
\hline Postural stability & $\begin{array}{l}\text { Extreme or bizarre responses to minimal } \\
\text { displacement }\end{array}$ & Initially minimal, progress with time \\
\hline Other neurologic features & False weakness, nonanatomic sensory loss & Usually absent \\
\hline Psychiatric symptoms & $\begin{array}{l}\text { Varied, usually evident but accurate definition } \\
\text { not always possible }\end{array}$ & May have depression as a part of disease \\
\hline Secondary gain & Litigation, compensation are usually present & Usually absent \\
\hline
\end{tabular}

Table 4: Different clinical tests for psychogenic motor deficit and their clinical implications

\begin{tabular}{ll}
\hline Clinical tests & Clinical implications \\
\hline Spinal Injuries Center Test & For psychogenic lower limbs weakness \\
Barre's Sign & For psychogenic hemiplegia. \\
Babinski Thigh-Trunk Test & For psychogenic hemiplegia and paraplegia. \\
Hoover's Test & For psychogenic hemiplegia. As with Barré's test, Hoover's test \\
& is of no use in the evaluation of paraplegia. \\
Monrad-Krohn's cough test & For psychogenic arm monoparesis. \\
Double-crossed-arm pull test & For psychogenic arm monoparesis. \\
Make-a-fist test & For psychogenic wrist drop. \\
Reversed hands test & For psychogenic hand paralysis. \\
Backward displacement test & For psychogenic foot drop. \\
Raimiste's leg adduction-abduction synkinesis & For psychogenic hemiplegia and leg monoparesis \\
\hline
\end{tabular}

\section{Babinski Thigh-Trunk Test}

To make an attempt to sit from a supine position, the natural tendency of the lower extremities is to tense in extension by contracting the gluteal muscles so as to provide a stable base. In true hemiplegia, the affected lower extremity will involuntarily exhibit thigh flexion when attempted to sit, because the gluteal muscles are weak and has no extensor tone. In paraplegia, both extremities will exhibit thigh flexion. The patient with psychogenic paralysis will typically maintain both lower extremities in extension position when he attempts to sit, reflexively firing the gluteal muscles. ${ }^{21}$

\section{Hoover's Test}

In the evaluation of hemiparesis, asking the patient to be in supine position, the examiner lifts the patient's legs slightly up from the examination table. While the examiner puts his hands below the heels, the patient is asked to lift the affected extremity; the absence of downward heel pressure in the contralateral (uninvolved) limb is indicative of apathy of intent (Fig. 1). As with Barré's test, Hoover's test is not useful in the evaluation of paraplegia. ${ }^{20}$

\section{Monrad-Krohn's Cough Test}

To identify psychogenic paralysis of the arm, the examiner stands behind the patient and grasps the bilateral latissimus dorsi muscles

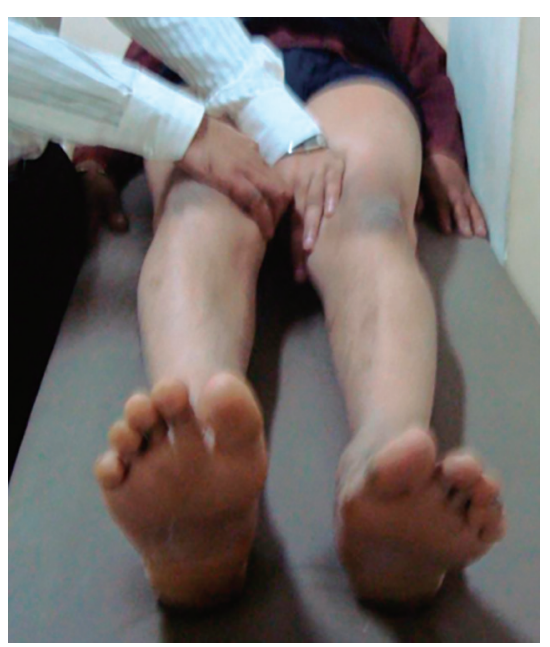

Fig. 1: Raimiste's leg adduction-abduction synkinesis

between the thumb and fingers of the right and left hands. The examiner asks the patient to cough forcefully. Both latissimus dorsi muscles synkinetically contact strongly, thus establishing the integrity of the motor pathway through the brachial plexus. 


\section{Double Crossed Arm Pull Test}

When required to use both sides simultaneously and unexpectedly, the psychogenic patient will generally inadvertently contract the putatively weak side along with the normal side. Patient remains upright with forearms crossed and flexed. If the patient's arm is completely paralyzed, it will be hold in place. While holding the patient's forearm, patient asks to pull back strongly away from examiner. Usually the patient braces the paretic and nonparetic arms when pulling back.

\section{Make a Fist Test}

To distinguish a psychogenic wrist drop from a radial palsy, the patient is asked to extend the arm out straight. The putatively paralyzed wrist hangs limply. The patient is instructed to suddenly make a strong fist. If intact, the putatively paralyzed wrist extensors automatically cock the hand into the "anatomic position" when the patient makes a fist.

\section{Raimiste's Leg Adduction-abduction Synkinesis}

With the patient recumbent, place hands on the patient's knees and the patient to adduct the legs together strongly. Examiner has to hold hands in place in opposition to the patient's action. The patient usually braces the putatively paralyzed limb in automatic opposition to the action of the intact limb (Fig. 2). Similarly, patient is asked to press the legs apart strongly against resistance. The putatively paralyzed limb usually will abduct in automatic position.

Difference between psychogenic and organic paraplegia is discussed in Table 5.

\section{Pharmacological Intervention}

Hypnotic sedatives have been used in the identification of psychogenic paralysis. The resolution after the administration of Sodium amytal has been reported in many case studies. ${ }^{22}$ This short-acting barbiturate has sedative, hypnotic, and anticonvulsant properties. The intravenous administration of $8 \mathrm{~mL}$ of $2.5 \%$ solution administered over 15 to 20 minutes has been shown to provide an immediate, effective, and lasting cure of psychogenic paralytic symptoms. It is hypothesized that dissociative medication and serial

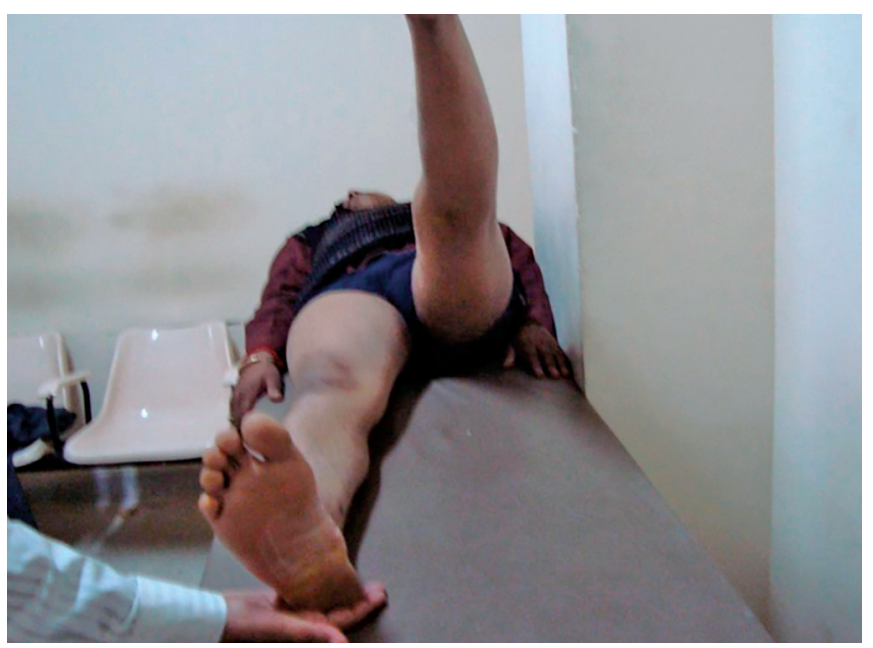

Fig. 2: Hoovers test

interviews will facilitate the recognition and resolution of symptoms by resolving subconscious (unrealized) conflicts.

\section{Nonepileptic Psychogenic Seizure (NEPS)}

Nonepileptic psychogenic seizurehas been defined as psychologically determined clinical event that resemble epileptic attack but is not associated with paroxysmal physiologic cerebral dysfunction. ${ }^{23}$ NEPS may constitute up to $20 \%$ of patients at epilepsy referral centers. ${ }^{24}$ To diagnose and treat NEPSis still challenging to the physicians.

\section{Etiology}

NEPSmay be due to reaction to a specific event. It may an expression of dependency or a wish or may show some attention-seeking needs. Preceding history of sexual or physical abuse can be present. ${ }^{24}$ Depression is common and suicide attempts have been reported. ${ }^{25}$ Tongue biting and other self-injurious behavior as well as incontinence during NEPS episodes predicts a higher likelihood of suicide attempts. ${ }^{25}$

Table 5: Differentiation of psychogenic and organic paraplegia

\begin{tabular}{lll}
\hline & Nonorganic paraplegia & Organic paraplegia \\
\hline $\begin{array}{l}\text { Ottitude to illness } \\
\text { Muscle stretch reflexes }\end{array}$ & Usually sudden after a stress & May evolve slowly or suddenly \\
Plantar response & May seem indifferent or histrionic & Appropriate concern \\
& Present and normal & Absent in spinal shock or very brisk \\
Clonus & Normal plantar flexion & $\begin{array}{l}\text { Dorsiflexion of great toe unless spinal shock } \\
\text { is present }\end{array}$ \\
Muscle tone & Absent or unsustained & Sustained \\
Abdominal/cremastric reflex & Normal & Flaccid acutely, then spastic \\
Umbilical migration & Present & Absent, depending on level \\
Sensory level & Absent & Upward migration if lesion affects T10 \\
& Extends horizontally around waist, variable, & Slants obliquely downward, constant border \\
Inadvertent leg use & differs from motor level & if lesion static \\
& May move legs inadvertently for postural & Does not move legs if the paraplegia is com- \\
Sphincter control & support, in sleep, or with Hoover's test & lete buy show flexor spasms \\
Anal wink reflex & Present & Lost \\
Magnetic resonance imaging, somatosensory & Normal but usually not needed & Lost in stage of spinal shock \\
evoked potential and cystometrogram & & Abnormal \\
\hline
\end{tabular}


Table 6: Behaviors to distinguish psychogenic nonepileptic and epileptic seizures

\begin{tabular}{|c|c|c|}
\hline Observation & Nonepileptic seizures & Epileptic seizures \\
\hline Situation onset & Occasional & Rare \\
\hline Gradual onset & Common & Rare \\
\hline Precipitated by stimuli (noise, light) & Occasional & May occur \\
\hline Motor activity & Prolonged, uncoordinated & $\begin{array}{l}\text { Automatisms, coordinated tonic } \\
\text { clonic activity }\end{array}$ \\
\hline Rhythmic pelvic movements & Pelvic thursting & Rare \\
\hline Opisthotonus & Occasional & Very rare \\
\hline Side to side head shaking & Occasional & Rare \\
\hline Tongue biting & Rare & $\begin{array}{l}\text { Common, mainly with tonic-clonic } \\
\text { seizures }\end{array}$ \\
\hline Prolonged ictal atonia & Ocassional & Very rare \\
\hline Postictal crying & Common & Rare \\
\hline Postictal confusion & Very rare & Common \\
\hline Closed mouth in tonic phase & Occasional & Very rare \\
\hline Vocalization during tonic-clonic phase & Occasional & Very rare \\
\hline Closed eyelids & Common & Very rare \\
\hline Duration & May be prolonged & Brief \\
\hline Resistance to eyelid opening papillary light reflex & Common & Very rare \\
\hline Reactivity during "unconsciousness" & Usually retained & Commonly absent \\
\hline Lack of cyanosis & Occasional & Very rare \\
\hline Rapid postictal reorientation & Common & Rare \\
\hline Stereotypy of attack & Common & Rare \\
\hline Diurnal variation & May be variable & Usually stereotypical \\
\hline Reproduction of seizures by suggestion & Daytime & Daytime or nocturnal \\
\hline \multirow[t]{2}{*}{ Triggers } & May occur & Very rare \\
\hline & Emotional disturbances & No \\
\hline
\end{tabular}

\section{Clinical Characteristics of NEPS}

NEPS's are episodes of altered movement, emotion, sensation, or experience, similar to epilepsy secondary to purely emotional causes. Patients are usually young adult but age may range from 4 to 77 years. ${ }^{27}$ NEPS episodes have been reported during sleep. However, EEGs show that the patient actually is awake before the episode begins. ${ }^{25} \mathrm{~A}$ variety ofsymptoms at onset like palpitation, malaise, choking, numbness, peripheral sensory disturbance, pain, odors or tastes, and visual hallucinations or distortions can be confused with an aura of epilepsy.

Some of the motor phenomena that are strongly associated with PNES are episodes with gradual onset or termination; events occurring during "pseudosleep", movements that are discontinous; irregular activity; side-to-side head movement; pelvic thrusting; opisthotonic posturing; stuttering; weeping; awareness that is preserved during motor activity; ${ }^{28 "}$ The evolution of the clonic jerks from fast and small-amplitude to slow and large-amplitude movements and the rapid contraction and slow relaxation of the true epileptic clonic jerks" are not usually seen in psychogenic seizures. The patient may have upward deviation of eyes, rapid tremorousmovements of the lids and resistance to eye-opening during NEPS episode. ${ }^{25}$

Apparently semipurposeful or purposeful behaviors like swallowing, mouthing, chewing, licking, smacking of the lips,looking about, picking up or moving objects, and undressing can occur and may mimic partial seizures. ${ }^{25}$ Behavior may be violent and includes biting, striking, kicking, slapping, pushing, obscene gesturing, clawing at the face or clothes, and directed rage. ${ }^{25}$ Vocalizations can occur during the episode. ${ }^{25}$ Autonomic changes can accompany psychogenic seizures and include light headedness; acral paresthesias; palpitations, tachycardia, dyspnea, or chest pain; headache; dysphoria or panic; fatigue or weakness; and muscle cramps, spasms, or tetany secondary to hyperventilation. ${ }^{25}$

The combination of widespread motor movements with subsequent ability to recall the details of the episode may be pointer toward diagnosis of NEPS. ${ }^{28}$ However, both focal and widespread unilateral motor seizures due to epilepsy can occur without loss of consciousness. Supplementary motor seizures characteristically involve both sides of the body, but patients often maintain awareness during the episode. ${ }^{25}$

Self-destructive behavioris seen in patients with psychogenic epilepsy which is suggested by urinary or fecal incontinance or both. ${ }^{25}$ Termination of the episode can be abrupt as in epilepsy or gradual. ${ }^{25}$ Psychogenic status or pseudostatus is an important and surprisingly common manifestation. The patients can present with a variety of symptoms, including all of those mentioned above. Intubation can be allowed by the patient. ${ }^{25}$ Complications due to emergency interventions for pseudostatus have included respiratory arrest, septicemia, pneumonia, urinary tract infection, cellulitis, and foot drop. Clinical features to distinguish psychogenic nonepileptic seizures from epileptic seizures are summarized in Table 6. 


\section{Diagnosis and Laboratory Testing of Psychogenic Seizures}

Diagnosis of NEPS is challenging but crucial. Delay in diagnosis has been reported from months to years with mean time of 7.2 years after initial manifestation to appropriate diagnosis. ${ }^{29}$ Delay may result in serious consequences like intubation and mechanical ventilation for pseudostatus to adverse effects of antiepileptic medications. ${ }^{30}$ Certain ictal features like gradual onset, bizarre and nonsynchronous movements are helpful in diagnosing psychogenic seizures, but none is absolute.

EEG: The combination of a normal EEG with an episode of loss of responsiveness is virtually diagnostic of a psychogenic seizure, though there are following important drawbacks.

An EEG can be normal during simple partial seizures especially from the frontal lobe. ${ }^{31}$ The use of additional scalp electrodes with anticonvulsant withdrawal, can improve ictal recordings. ${ }^{32}$ Focalor generalized slowing secondary to diffuse encephalopathy or a cortical lesion is notnecessarily an indication of epilepsy. ${ }^{25}$ Generalized epileptiform bursts in patients with drug withdrawl such as barbiturate withdrawal, artifacts or normal-variant patterns and definite epileptiform discharges in patients without epilepsy, or in asymptomatic relatives of patients with epilepsy can be confused as an evidence of epilepsy. ${ }^{25}$ Interictal epileptiform discharges did not exclude a diagnosis of psychogenic seizures. Syncope might cause loss of consciousness, or a fall and a short lasting episode of shaking of the limbs. The EEG might show rhythmic slow-wave discharges or EEG "flattening" of at the time of the episode. This is anon epilepti form EEG abnormality. Finally, it is difficult to comment on EEG that has many movement artifacts during ictal period and in such situation it may become difficult to comment on epileptiform discharges.

Long video-EEG monitoring documenting spontaneous and provoked typical episode is the "gold standard" test with advantage of simultaneous correlation of behavior and the EEG pattern. Recorded event can be shown to patient, family members or care givers so that they can compare with prior episodes. However, video EEG does not exclude the possibility of coexisting epilepsy and it can be misinterpreted or can mislead.

Serum prolactin: A several-fold increase in prolactin level relative to baselinewithin 15 to 30 minutes after the episode is suggestive of epileptic episode. Cut off level of $500 \mathrm{IU} / \mathrm{ml}$ for serum prolactin was found to be useful and value greater than this was reported in $90 \%$ of patients after generalized tonic clonic seizures and in $60 \%$ after complex partial seizures. ${ }^{33}$ Prolactin level elevation is more common after generalized tonic-clonic or temporal lobe seizures than frontal lobe or other simple partial seizures. ${ }^{34}$ Both false-positive and falsenegative results can occur. False positive result has been shown after syncope. ${ }^{35}$ Prolactin levels are not significantly increased after psychogenic seizures. Absenceof postictal prolactin elevation is not a reliable indicator of a psychogenic seizure.

\section{Seizure Induction Protocols}

Because episodes do not necessarily occur spontaneously in the laboratory, there has been wide interest in induction procedures. Compression of body parts, photic stimulation, verbal suggestion, placement of a tuning fork or moistened patches on the skin, and intravenous administration of saline or other placebos are the method utilized to provoke psychogenic seizures. ${ }^{25}$ Induction of an episode should be done with emotional support and dignity of the patient should be maintained. Induction method has similar importance as neurologic examination maneuvers that areutilized to elicit neurological signs has.

The concept of beneficence as applied to medical ethics dictates that the physician should not only respect and avoid harming a patient, but also should make positive contributions toward the patient's well-being and towards the removal of possible harmful conditions, maximizing the benefits and minimizing the risks of a particular treatment. ${ }^{36}$ An induction procedure has important contribution to this by avoiding the potential hazards of an inappropriate diagnosis and inappropriate treatments. It also facilitates the appropriate treatment for the patient's condition. In response to induction not every patient with psychogenic seizures will have an episode and in the course of an induction procedure it might be possible that an episode of epilepsy occurs. ${ }^{25}$

\section{Organic Disorders with Bizarre or Subtle Neurologic Manifestations often Mistaken for Psychogenic Illness}

Several neurological, metabolic or immunological disorders may have variable neurological presentations. If these symptoms vary in severity, inconsistency on repeated neurological examinations or if they have unexplainable presentations, they can mimic conversion or somatoform disorders. Porphyria, multiple sclerosis, myasthenia gravis, complex partial seizures and collagen vascular diseases are among the commonest mimickers. ${ }^{37}$ Early presentations of these disorders may be misinterpretated as a conversion or somatoform disorders and it may delay specific therapy of these organic disorders. One systematic review reported higher rate of misdiagnosis of conversion disorders before 1970 and consistently low rate of $4 \%$ after every decade since than. ${ }^{38}$ Commonly epilepsy, movement disorders and multiple sclerosis were misdiagnosed as conversion reaction. Possible causes of misdiagnosis were bizarre presenting symptoms and associated psychiatric history. It can cause devastating effect on outcome. Clinician should be more vigilant before considering unexplainable symptoms as nonorganic. In case of any doubt, patient should be thoroughly investigated.

\section{Treatment}

The literature regarding management of conversion disorders is scanty. Only few control trials have been conducted in this area. Most of the studies are conducted with small sample sizes and with inadequate control. The outcome measures (dependent variables) are also poorly defined in most of the studies. Most of the studies lack follow up sessions which are very important in the management of conversion disorders. Associated psychopathology such as depression and anxiety are not addressed in most of the studies. Patients diagnosed with conversion disorder frequently benefit from a team approach to treatment and from a combination of treatment modalities. A team approach is particularly beneficial if the patient has a history of abuse, or if he or she is being treated for a concurrent physical condition or illness.

\section{Explanation and Reassurance}

A good explanation of the symptoms toa patient with a conversion disorder is a prerequisite of successful further treatment. ${ }^{39}$ In explanation and discussion, terms such as psychogenic or functional should be avoided. These terms give impression of intentional act by patient. Scientific term like conversion disorder should be used and patient should be explained about the nature of its symptoms and role of psychosocial stressors. The manner of explanation should be empathetic. It is also difficult to explain and convince friends, 
family, and employers about the diagnosis. Neurologists, are usually not well verse with this entity and they do not know how to further pursue the patient. It can be simply explained to the patient that they do not have any neurological disease.

\section{Psychotherapy}

Psychodynamic psychotherapies are historically popular in the treatment of conversion disorders, but they are less used emperically. A recent $\mathrm{RCT}$ in patients with psychogenic nonepileptic seizures showed benefit from cognitive behavioral treatment (CBT) compared with standard therapy over a 6-month period. ${ }^{40}$ Group therapy, preferably in conjunction with concurrent individual therapy, offers advantages of reinforcing psycho educational concepts, while providing the opportunity for patients to learn from each other and also help each other. Two noncontrolled studies have reported benefit of psychotherapy to patients having nonepileptic seizures. ${ }^{41,42}$ Multidisciplinary in patient treatment may be preferred to patients with severe and prolonged symptoms, ${ }^{43-45}$ but such resources are not available for all patients.

Psychodynamic psychotherapy is sometimes used with children and adolescents to help them gain insight for their symptoms. Family therapy is often recommended for younger patients whose symptoms may be related to some family issues. Group therapy appears to be particularly useful in helping adolescents to learn social skills and copying strategies, and to decrease their dependency on their families. Recently a Cochrane review was unable to draw a conclusion about potential benefits or harms of psychosocial interventions for conversion disorders from the present studies. ${ }^{46}$ This emphasizes on the need of more scientific studies and randomized controlled trials to elicit role of psychosocial therapies.

\section{Hypnosis}

Hypnosis has been advocated for the treatment of conversionsymptoms since the time of Charcot, Janet, andFreud. Neuroimaging data support the idea that conversionsymptoms and hypnosis involve common neurological pathways, and the high hypnotizability of these patients promotes the use of hypnosis in the treatment. Forty-four outpatients who were suffering from conversion disorder were randomly assigned to a hypnosis or a waiting-list condition. It was seen that there was more improvement in hypnosis-condition patients as compared to baseline and waiting list controls. ${ }^{47}$ Another study comparing a comprehensive treatment program comprising intensive group therapy, social skills training, creative therapy, sports therapy, and physical therapy with or without hypnosis showed no added benefit from hypnosis for resolving conversion symptoms and no predictive value of hypnotizability for treatment outcome. ${ }^{44}$ Hypnosis can be a useful adjunctive treatment, but it is not essential for improvement. A comprehensive approach is likely to be the most effective. Hypnosis without other forms of psychiatric treatment may decrease conversion symptoms but have less impact on overall psychopathology.

\section{Pharmacotherapy}

In the absence of sufficient evidence based data and recommendations, the current practice is to use medications appropriate for the comorbid psychiatric and somatic symptoms. Anecdotal studies report improvementwith selective serotonin reuptake inhibitors (SSRIs), betablockers, analgesics, and benzodiazepines. ${ }^{48}$ An opentrial of antidepressants in patients with psychogenic movement disorder and recent or current depression also showed that class of medications to be effective in reducing conversion symptoms. ${ }^{49}$ Recently a pilot, double-blind, randomized, placebo-controlled trial showed Class II evidence that flexible-dose sertaline up to a maximum dose of $200 \mathrm{mg}$ is associated with a nonsignificant reduction in PNES rate compared with a placebo control arm (risk ratio $0.51,95 \%$ confidence interval $0.25-1.05, p=0.29$ ), adjusting for differences at baseline. ${ }^{50}$ If patient is on antiepileptic drugs and diagnosis is likely to be nonepiletic psychogenic seizure, antiepileptic drugs should be stopped in view of their side effects and no therapeutic role. Barbiturates and benzodiazepines should be tapered gradually to avoid withdrawal symptoms. Some antiepileptic drugs have mood stabilizing property like Valproic acid and Lamotrigine, these may be continued if patient has associated bipolar mood disorder. In one study, randomized controlled trial was done to compare effect of immediate versus delayed withdrawal of antiepileptic drugs on outcome, There was a significant reduction in spell frequency and use of rescue medication from baseline to 9 months in the immediate withdrawal group compared to delayed withdrawal group. Emergency health care utilization dropped to zero in both groups by the end of the study. ${ }^{51}$ No controlled studies have evaluated atypical antipsychotics for the treatment of conversion reactions, particularly in the absence of frank paranoia or psychosis. Reports of the benefits of antipsychotic medications in conversion reactions ${ }^{52-54}$ are anecdotal.

\section{Transcranial Magnetic Stimulation}

More recent anecdotal reports showed benefit of transcranial magnetic stimulation in refractory conversion paralysis ${ }^{55}$ and somatization associated with posttraumatic stress disorder. ${ }^{56}$ Frontal-subcortical circuits disruption in these disorders as shown by functional neuroimaging supports the use of transcranial magnetic stimulation in coversion disorders. Future procedures those directly targeting frontal-subcortical circuit will ultimately benefit patients with conversion disorders.

\section{The Patient Who Does Not Improve}

All patients with conversion disorder do not respond in meaningful manner. Despite best efforts, if patients does not improve, they can be ensured that best treatment has been given to them for the time being and their symptoms might improve in future if they continue the medication. For time being, therapy should be targeted on present symptomatology like anxiety, depression or other mood disorders. Patient should be explored for other psychosocial stressors or other perpetuating factors. Such patients should be under close follow-up as few organic diseases may mimic conversion disorders as highlighted previously. It is important to protect them from unnecessary investigations and treatment and consideration for referral to specialist center if needed.

\section{Role of Neurologist in Management of Conversion Disorders}

Traditionally, neurologists don't play significant role in management of conversion disorders and refer them to a psychiatrist. However, neurologists can play an important role with further visits to reinforce the explanation and rationale for the diagnosis. After gaining trust in the treating neurologist, it becomes relatively easy to discuss any associated psychological factors with the patient and, 
if needed, a referral can be done to a psychiatrist /psychologist. Significant number of all neurology outpatients have functional symptoms, so it is unlikely to access specialist psychological treatment by all. Psychiatric/psychosocial treatment is not needed for most of the patients and patients with mild symptoms can be managed simply by conversation, explanation and reassurance. If there is no response to appropriate therapy or if there is any doubt in diagnosis of conversion disorder, neurologist plays an important role to look for any missed organic disorder.

\section{Course and Prognosis}

Between $50 \%$ and $90 \%$ of the patients with conversion disorder exhibit short-term resolution of symptoms after reassurance, but as many as $25 \%$ of these responders relapse or develop new conversion symptoms over time. ${ }^{57,58}$ Worst prognostic factors are longer duration of symptoms, psychiatric comorbidity, subacute presentation, and tremor. Among patients with nonepileptic seizures, even those with symptomatic improvement may remain disabled. ${ }^{59}$ In one outcome study of 56 such patients, only half of the patients had a resolution of nonepileptic seizures, a mean of 1.5 years after diagnosis, and many still exhibited depressive symptoms, suicidal ideation, and suicide attempts. A patient's perception of good health and occupational functioning is correlated with resolution, which suggests that interventions that focus on improving functioning and self-esteem could aid treatment. ${ }^{60}$

In conclusion, this review has limitations as every aspect of conversion disorders cannot be highlighted. Conversion disorders have wide spectrum of manifestation ranging from sensorimotor deficit to movement disorders to nonepileptic seizures. Management of conversion disorders is more challenging as their diagnosis. No evidence based guideline is available as yet. Treatment is mainly targeted to explanation, reassurance and treating associated comorbidities. Relapses and other psychiatric manifestation are common.

\section{References}

1. Wenegrat B. 2001. Theatre of disorder - patients, doctors and the construction of illness. Oxford: Oxford Univ Pr.

2. Breuer J, Freud S Studies in Hysteria.1895.

3. American Psychiatric Association: Diagnostic and Statistical Manual of Mental Disorders, 4th Edition, Text Revision (DSM-IV-TR). Washington, DC, American Psychiatric Publishing, 2000.

4. Heruti RJ. Conversion motor paralysis disorder. South Med J 2006;99:327-328.

5. Lempert T, Dietrich M, Huppert D, Brandt T. Psychogenic disorders in neurology: frequency and clinical spectrum. Acta Neurol Scand 1990;82:335-340.

6. Putnam FW. Conversion symptoms. Movement disorders in neurology and neuropsychiatry. Boston, Blackwell Scientific Publications 1992. pp.430-437.

7. Lazare A. Current concepts in psychiatry ; Conversion symptoms. N Engl J Med 1981;05:745-748.

8. Fahn S, Williams D. Psychogenic dystonia. Adv Neurol 1988;50:431455.

9. Shill H, Gerber P. Evaluation of clinical diagnostic criteria for psychogenic movement disorders. Mov Disord. 2006;21:1163-1168.

10. Gupta A, Lang AE. Psychogenic movement disorders. CurrOpin Neurol 2009; 22(4): 430-436.

11. Weiner W, Lang A. Idiopathic torsion dystonia. Weiner WJ, Lang A (eds): A comprehensive survey. New York, Futura Publishing Co 1989. pp.221-256.

12. Williams DT, Ford B, Fahn S. Phenomenology and psychopathology related to psychogenic movement disorders. Adv Neurol. 1995;65:231-257.
13. Mary Ann Thenganatt and Joseph Jankovic; Psychogenic Tremor: A Video Guide to Its Distinguishing Features; Tremor and other hyperkinetic movements; 2014; 4: 253; doi: 10.7916/D8FJ2F0Q.

14. Kim YJ, Pakiam AS, Lang AE. Historical and Clinical Features of Psychogenic Tremor:a Review of 70 Cases. Can. J. Neurol. Sci 1999;26:190-195.

15. Monday K, Jankovic J. Psychogenic myoclonus. Neurology 1993;43:349-352.

16. Lang AE, Koller WC, Fahn S. Psychogenic parkinsonism. Arch Neurol 1995;52:802-810.

17. Binzer MN, Kullgren G. Psychogenic paralysis: A prospective study. UgeskrLaeger. 2000;162:5632-5636.

18. Hill E, Haydel M. Conversion disorder presenting as hemiplegia and hemianesthesia with loss of neurologic reflexes: a case report. South Med J 2006;99:380-382.

19. Baker JH, Silver JR. Hysterical paraplegia. J Neurol Neurosurg Psychiatry 1987;50:375-382.

20. Yugue I, Shiba K, Ueta T, Iwamoto Y. A new clinical evaluation for hysterical paralysis. Spine 2004;29:1910-1913.

21. Okun MS, Koehler PJ. Babinski's clinical differentiation of organic paralysis from hysterical paralysis: effect on US neurology. Arch Neurol 2004;61:778-783

22. Garofalo ML. The diagnosis and treatment of hysterical paralyses by the intravenous administration of pentothal sodium - case reports: 1942. Conn Med 1992;56:159-160.

23. Andriola MR, Ettinger AB. Pseudoseizures and other nonepileptic paroxysmal disorders in children and adolescents. Neurology 1999;53:S89-S95.

24. Alper K, Devinsky O, Perrine K, Vazquez B, Luciano D. Non-epileptic seizures and childhood sexual and physical abuse. Neurology 1993;43:1950-1953.

25. Ronald P. Lesser. Psychogenic Seizures. Neurology. June 01, 1996;46 (6).

26. Boddea NMG, Brooksd JL, Bakerd GA, Boonab PAJM, Hendriksena JGM, Muldera OG, AP Aldenkampac. Psychogenic non-epileptic seizures-Definition, etiology, treatment and prognostic issues: A critical review. Volume 18, Issue 8, October 2009, Pages 543-553.

27. Edwin Peguero, BasselAbou-Khalil Self-Injury and Incontinence in Psychogenic Seizures. Epilepsia 36(6):586-91. July 1995.

28. J. Stephen Huff; Najib Murr. Seizure, Pseudoseizures. Treasure Island (FL): StatPearls Publishing; 2018 Jan-.

29. Reuber M, Elger CE. Psychogenic nonepileptic seizures: review and update. Epilepsy Behav. 2003;4(3):205-216. Benbadis SR. Nonepileptic behavioral disorders: diagnosis and treatment. Continuum (MinneapMinn) 2013;19(3 Epilepsy):715-729.

30. Wesley T. Kerr, Ph.D., ${ }^{1,2}$ Emily A. Janio, ${ }^{2}$ Justine M. Le, B.S., ${ }^{2}$ et al. Diagnostic delay in psychogenic seizures and the association with anti-seizure medication trials. Seizure. 2016 Aug; 40: 123-126.

31. Bromfield EB, Cavazos JE, Sirven JI. Clinical Epilepsy. An Introduction to Epilepsy [Internet]. American Epilepsy Society; 2006.

32. M. G. Marciani, J. Gotman. Effects of Drug Withdrawal on Location of Seizure Onset. Epilepsia. August 19 https://doi. org/10.1111/j.1528-1157.1986.tb03562.x

33. Om Shankar Chaurasiya, Parul Gupta, Anil Kaushik, Rajesh Yadav. Usefulness of Serum Prolactin in Differentiating Epileptic and Pseudoseizures in Children. People's Journal of Scientific Research . Vol. 6(1), Jan. 2013.

34. AbuhuziefaAbubakr, and Ilse Wambacq, Diagnostic value of serum prolactin levels in PNES in the epilepsy monitoring unit.

35. Oribe E, Amini R, Nissenbaum E, et al. Serum prolactin concentrations are elevated following syncope. Neurology 1996;47:60-62.

36. Beauchamp TL, Childress JE. Principles of biomedical ethics. New York: Oxford University Press, 1979.

37. DePaulo JR, Folstein MF. Psychiatric disturbances in neurological patients: detection, recognition and hospital course. Ann Neurol 1978;4:225-228.

38. Stone J, Smyth R, Carson A, et al. Systematic review of misdiagnosis of conversion symptoms and "hysteria". BMJ 2005;331:989. 
39. Carton S, Thompson PJ, Duncan JS. Non-epileptic seizures: patients' understanding and reaction to the diagnosis and impact on outcome. Seizure 2003; 12:287-294.

40. Goldstein LH, Chalder T, Chigwedere C, et al. Cognitive-behavioral therapy for psychogenic nonepileptic seizures: a pilot RCT. Neurology 2010;74(24):1986-1994.

41. Zaroff CM, Myers L, Barr WB, Luciano D, Devinsky O: Group psychoeducation as treatment for psychological nonepileptic seizures. Epilepsy Behav 2004; 5:587-592.

42. Wittenberg D, Michaels J, Ford C, Bullock K, Barry JJ: Group psychotherapy for patients with non-epileptic seizures: a pilot study (abstract). Epilepsia 2004; 45 (suppl 7):57-58.

43. McDade G, Brown SW: Non-epileptic seizures: management and predictive factors of outcome. Seizure 1992; 1:7-10.

44. Kim CM, Barry JJ, Zeifert PA: The use of inpatient medical psychiatric treatment for nonepileptic events (abstract). Epilepsia 1998; 39(suppl):242-243.

45. Moene FC, Spinhoven P, Hoogduin KA, van Dyck R: A randomized controlled clinical trial on the additional effect of hypnosis in a comprehensive treatment programme for in-patients with conversion disorder of the motor type. PsychotherPsychosom 2002; 71:66-76.

46. Ruddy R, House A. Psychosocial interventions for conversion disorder. Cochrane Database of Systematic Reviews 2009, Issue 1.

47. Moene FC, Spinhoven P, Hoogduin KA, van Dyck R: A randomized controlled clinical trial of a hypnosis-based treatment for patients with conversion disorder, motor type. Int J Clin Exp Hypn 2003; 51:29-50.

48. LaFrance WC Jr, Devinsky O: The treatment of nonepileptic seizures: historical perspectives and future directions. Epilepsia 2004; 2(45 suppl):15-21.

49. Voon V, Lang AE: Antidepressant treatment outcomes of psychogenic movement disorder. J Clin Psychiatry 2005; 66:1529-1534.
50. LaFrance WC Jr, Keitner GI, Papandonatos GD, Blum AS, Machan JT, Ryan CE, Miller IW. Pilot pharmacologic randomized controlled trial for psychogenic nonepileptic seizure. Neurology 2010;75(13):11661173.

51. Oto M, Espie CA, Duncan R. An exploratory randomized controlled trial of immediate versus delayed withdrawal of antiepileptic drugs in patients with psychogenic nonepileptic attacks (PNEAs). Epilepsia.2010;51(10):1994-1999.

52. Drake ME Jr, Pakalnis A, Phillips BB: Neuropsychological and psychiatric correlates of intractable pseudoseizures. Seizure 1992 1:11-13.

53. Marazziti D, Dell'Osso B: Effectiveness of risperidone in psychogenic stiff neck. CNS Spect 2005; 10:443-444.

54. Persinger MA: Seizure suggestibility may not be an exclusive differential indicator between psychogenic and partial complex seizures: the presence of a third factor. Seizure 1994; 3:215-219.

55. Schonfeldt-Lecuona C, Connemann BJ, Spitzer M, Herwig U: Transcranial magnetic stimulation in the reversal of motor conversion disorder. Psychotherapy Psychosom 2003; 72:286-288.

56. Grisaru N, Amir M, Cohen H, Kaplan Z: Effect of transcranial magnetic stimulation in posttraumatic stress disorder: a preliminary study. Biol Psychiatry 1998; 44:52-55.

57. Hafeiz HB: Hysterical conversion: a prognostic study. Br J Psychiatry 1980; 136:548-551.

58. Baker JH, Silver JR: Hysterical paraplegia. J Neurol Neurosurg Psychiatry 1987; 50:375-382.

59. Pestana EM, Foldvary-Schaefer N, Marsillio D, Morris HH III: Quality of life in patients with psychogenic seizures. Neurology 2003; 60 (suppl 1): A355.

60. Ettinger AB, Devinsky O, Weisbrot DM, Ramakrishna RK, Goyal A: A comprehensive profile of clinical, psychiatric, and psychosocial characteristics of patients with psychogenic nonepileptic seizures. Epilepsia 1999; 40:1292-1298. 